\title{
Intermittent criticality in the long-range connective sandpile (LRCS) model
}

\author{
Chien-chih Chen ${ }^{\mathrm{a}, *}$, Ya-Ting Lee ${ }^{\mathrm{a}}$, Ling-Yun Chiao ${ }^{\mathrm{b}}$ \\ a Department of Earth Sciences and Graduate Institute of Geophysics, National Central University, Jhongli 320, Taiwan, ROC \\ ${ }^{\mathrm{b}}$ Institute of Oceanography, National Taiwan University, Taipei 106, Taiwan, ROC
}

\section{A R T I C L E I N F O}

\section{Article history:}

Received 17 January 2008

Received in revised form 2 April 2008

Accepted 7 April 2008

Available online 25 April 2008

Communicated by A.R. Bishop

\section{PACS:}

45.70.Ht

45.70.Cc

$05.65 .+b$

05.70.Ln

Keywords:

Sandpile model

Self-organized criticality

Small-world network

Long-range connection

Seismicity

\begin{abstract}
A B S T R A C T
We here propose a long-range connective sandpile model with variable connection probability $P_{c}$ which has an important impact on the slope of the power-law frequency-size distribution of avalanches. The long-range connection probability $P_{c}$ is changed according to an explicit function of the size of the latest event, although the evolution rule of $P_{c}$ may be different in various physical systems. Such version of the sandpile model demonstrates large fluctuations in the dynamical variable $\langle Z\rangle(t)$ (the spatially averaged amount of grains retained within the grid at each time step), indicating the state of intermittent criticality in the system. Many researches have suggested that the earthquake fault system is an intermittent criticality system, which would imply some level of statistical predictability of great events. Our modified sandpile model thus provides a testing ground for many proposed precursory measures related to great earthquakes.
\end{abstract}

(C) 2008 Elsevier B.V. All rights reserved.

\section{Introduction}

In analogy to the study of critical phase transitions in statistical physics, there had been attempts to approach earthquakes in the context of a critical phenomenon [1-6]. The hope of revealing new insights that would not be possible in the conventional perspective gets even higher since the concept of self-organized criticality was introduced in Bak et al. [7]. Earthquakes have then been treated as an example of this phenomenon in nature [8-10], and the empirical Gutenberg-Richter law has been suggested to be the manifestation of the self-organized critical state of the collective dynamics of the earthquake faults. In addition to self-organized criticality in which the system is always at or near critical state, intermittent criticality, in which a system repeatedly approaches and retreats from a critical state, had also been demonstrated in the work of Sammis and Smith [11]. One center manifestation from it is the time-dependent variations in the frequency-magnitude distribution during a seismic cycle. To differentiate whether the Earth's crust is in a single self-organized critical state, or a composite intermittent

\footnotetext{
* Corresponding author at: Institute of Geophysics, National Central University, No. 300, Jhongda Rd., Jhongli, 320, Taiwan, ROC. Tel.: +886 34227151 65653; fax: +8863422 2044 .

E-mail address: chencc@ncu.edu.tw (C.-c. Chen).
}

criticality would be of fundamental importance for understanding the statistics and the physics of earthquakes and be potentially enlightening in addressing the question of whether earthquakes can be forecast [12-15].

The concept of intermittent criticality is based on the hypothesis that a great earthquake on the fault network cannot occur until regional criticality threshold has been reached and stress is consequently correlated at all length scales up to the size of the region. This great event then destroys the overall regional criticality on its associated network. After a period of relative quiescence following the great event, the dynamical process of the earthquake fault system repeats by reloading energy and rebuilding correlation lengths towards the criticality that leads to the next great event. The situation of intermittent criticality would imply some level of statistical predictability of great events in the population dynamics whereas, in the case of self-organized criticality, perpetually large stress correlations reduce the degree of predictability of great events. Models exhibiting the intermittent criticality state include, for example, the lossy and structurally heterogeneous automaton in Sammis and Smith [11], the small-dissipation and large-neighborhood sandpile model in Castellaro and Mulargia [13], and the discrete model of faults with strength heterogeneities in Ben-Zion et al. [16] as well. Here we would like to propose an alternative variant of the sandpile model with randomly internal connections [17] to demonstrate 
the state of intermittent criticality. Comparison between different models will be also discussed in the end of this Letter.

\section{Sandpile model with variable long-range connective probability}

We build our sandpile model by a very simple set of rules that is very similar to the original one [7]. For a square grid of $L$ by $L$ cells, we randomly throw sands, one at a time, onto the grid. In the original sandpile model, once the total amount of the accumulated sands within a single cell reaches the threshold amount of 4 , they will be redistributed to the four adjacent cells (the nearest neighbors) or lost off the edge of the grid. All the cells receiving grains from their neighbors will be checked, and the redistribution would be continued further away if any one of them reaches consequently over the threshold too. For each throw of new grains, the redistribution proceeds until none of the meshes receiving new grains exceeds the threshold. The total amount of cells involved in the redistribution process initiated by a single throw is defined to be the size of the toppling event.

To implement randomly internal connections in the sandpile model the redistribution process has been modified. Our modified rule of randomly internal connections is very similar to the implementation of Watts and Strogatz [18]. For any particular cell, when the accumulated grains exceed the threshold and redistribution occurs, one of the original nearest neighbor connections confronts a chance with the connective probability $P_{c}, 0<P_{c}<1$, of redirecting to a randomly chosen, distant cell and so the original connection is replaced by a randomly chosen mesh that might be faraway from the toppling cell. We may call such version of sandpile model the long-range connective sandpile (LRCS) model. Apparently, when $P_{c}=0$, the LRCS model reduced to the original nearest neighbor sandpile model by Bak et al. [7]. It has been found the connective probability $P_{c}$ has a significant impact on the scaling exponents of the power-law frequency-size distributions in our modified LRCS model [17].

According to Chen et al. [17], lower $P_{c}$ implies higher activity of total toppling and lower scaling exponent of the powerlaw frequency-size distribution. We thus presume that a possible mechanism of $P_{c}$ evolution is to reflect the contemporary toppling size, or the topographic change. The rationale is that when the system is activated by a large event, there is higher probability to establish long-range random connection due to, for example, increases of system permeability, fingerling channels of pore pressure or the dynamic triggering of seismic waves. Particularly, seismicity increased in Long Valley Caldera following the $M_{w}$ (moment magnitude) $=7.92002$ Denali Fault earthquake, $M_{w}=7.11999$ Hector Mine earthquake and $M_{w}=7.31992$ Landers earthquake. The three main shocks were 3460, 400, and 440 $\mathrm{km}$ from Long Valley, respectively. As pointed out by previous studies, e.g., [27], in these long-range triggering cases, the stress perturbation of seismic waves is the immediate cause of the triggered earthquakes. Although remote triggered seismicity has been robustly documented for several large events and has now been seen up to $4000 \mathrm{~km}$ from the main shock, the exact mechanism for the dynamic triggering remains unclear. Whereas when the system is cooled down with less activity, it gets into a healing stage that the tectonic loading gradually shuts off most of the long-range connections and thus reduces the $P_{c}$.

Consider the topographic height of the sandpile at the time step $i$ is $Z_{i}(x)$. At the next time step $i+1$, due to the throw of single grain on the grid, it changes from $Z_{i}(x)$ to $Z_{i+1}(x)$. Therefore, total change in the topographic height of the sandpile is $\Delta Z_{i+1}=$ $\sum_{x_{l}}\left|Z_{i+1}\left(x_{l}\right)-Z_{i}\left(x_{l}\right)\right|$. Usually, on a square $L$ by $L$ grid, $\Delta Z$ ranges from 1 , which means no toppling occurs, to $\alpha L^{2}$ ( $\alpha \cong 1.25$ ). We then simply define $P_{c}=\left(\Delta Z / \alpha L^{2}\right)^{2}$. It is very likely that there are many other different ways to define $P_{c}$ for different physical systems. To our end of demonstrating intermittent criticality in the sandpile model, we have found that the exact choice of formulation for $P_{c}$ is not crucial.

We have calculated the spatially averaged amount of grains retained within the grid at each time step, i.e., $\langle Z\rangle(t)$, to indicate the temporal evolution of the dynamical behavior of the system. Shown in Fig. 1 is an example of simulating result for the LRCS model with variable $P_{c}$ (green line), 2000 computer generations extracted from a series of experiments each with $10^{5}$ throws of single grain on a square $50 \times 50$ grid. In each experiment, $P_{c}$ starts with 0 and then changes with an evolutional rule as described above. For comparison, also shown in Fig. 1 are two cases with constant $P_{c}=0$ (blue solid line) and $P_{c}=1$ (blue dotted line), respectively. The beginning, transient thousands of iterations, where $\langle Z\rangle(t)$ increases linearly, have been ignored. For the original nearest neighbor sandpile model, i.e., the case of $P_{c}=0,\langle Z\rangle(t)$ fluctuates about an average value of about 2.1, whereas a notable decrease of the average of $\langle Z\rangle(t)$, which is about 1.8 , could be found for the LRCS model with constant $P_{C}=1$. Such statistically stationary value of $\langle Z\rangle(t)$ is recognized as a manifestation of the self-organized critical state [8]. The power-law frequency-size distribution (Fig. 2) is, on the other hand, another expression of the self-organized critical state $[7,19,20]$.

Unlike the pattern for a fixed implementation of $P_{c}$ (blue lines in Fig. 1), the dynamic variable of $\langle Z\rangle(t)$ in the LRCS model with self-evolved $P_{c}$ is often punctuated towards a smaller value by a large event while its baseline approximately corresponds to the average of $\langle Z\rangle(t)$ in the case of constant zero $P_{c}$. For the LRCS model with self-evolved $P_{c}$ (green line in Fig. 1 ), large fluctuation in $\langle Z\rangle(t)$ is an important and interesting feature mimicking the state of intermittent criticality. It is noted that, in a long run, the power-law frequency-size distribution of avalanches in our LRCS model with variable $P_{c}$ (Fig. 2) is not disrupted. In other words, it seems not possible through the aggregately long-term frequencysize distribution of events to distinguish the intermittent critical state in our LRCS model from the self-organized criticality in the original Bak-Tang-Wiesenfeld-type sandpile model [7]. According to such observation, we thus raise the important and fundamental question about whether it is possible to invent relevant measure $[15,16]$ to detect precursors of great events in the intermittent criticality system of our LRCS model if regional seismicity can be verified to behave as so. One of the widely reported precursors is the decline in the slope of the Gutenberg-Richter power law for frequency-magnitude distribution of earthquakes [17]. Since the scaling exponent in the power-law frequency-size distribution of avalanches mimics the slope of the Gutenberg-Richter power law of earthquakes, we have calculated the exponents of the frequencysize distributions with many short-term samples consisting of 500 avalanches. We have found that the fluctuation in those short-term exponents of the frequency-size distributions for our LRCS model with variable $P_{c}$ is the double to triple of the exponent fluctuation for the BTW sandpile model. Moreover, in the adaptable LRCS model, we have also found that the power-law slopes frequently decrease before large events. We will postpone to future work the detailed analysis of the precursory phenomenon in our LRCS model.

\section{Discussion}

Thriving models based on cellular automata have been recently employed for understanding the earthquake physics $[8,11$, 13,21-23]. One type of simple cellular automata models is mainly the Bak-Tang-Wiesenfeld-type sandpile model [7]. In the BTW sandpile model, the self-organized criticality, characterized by the 


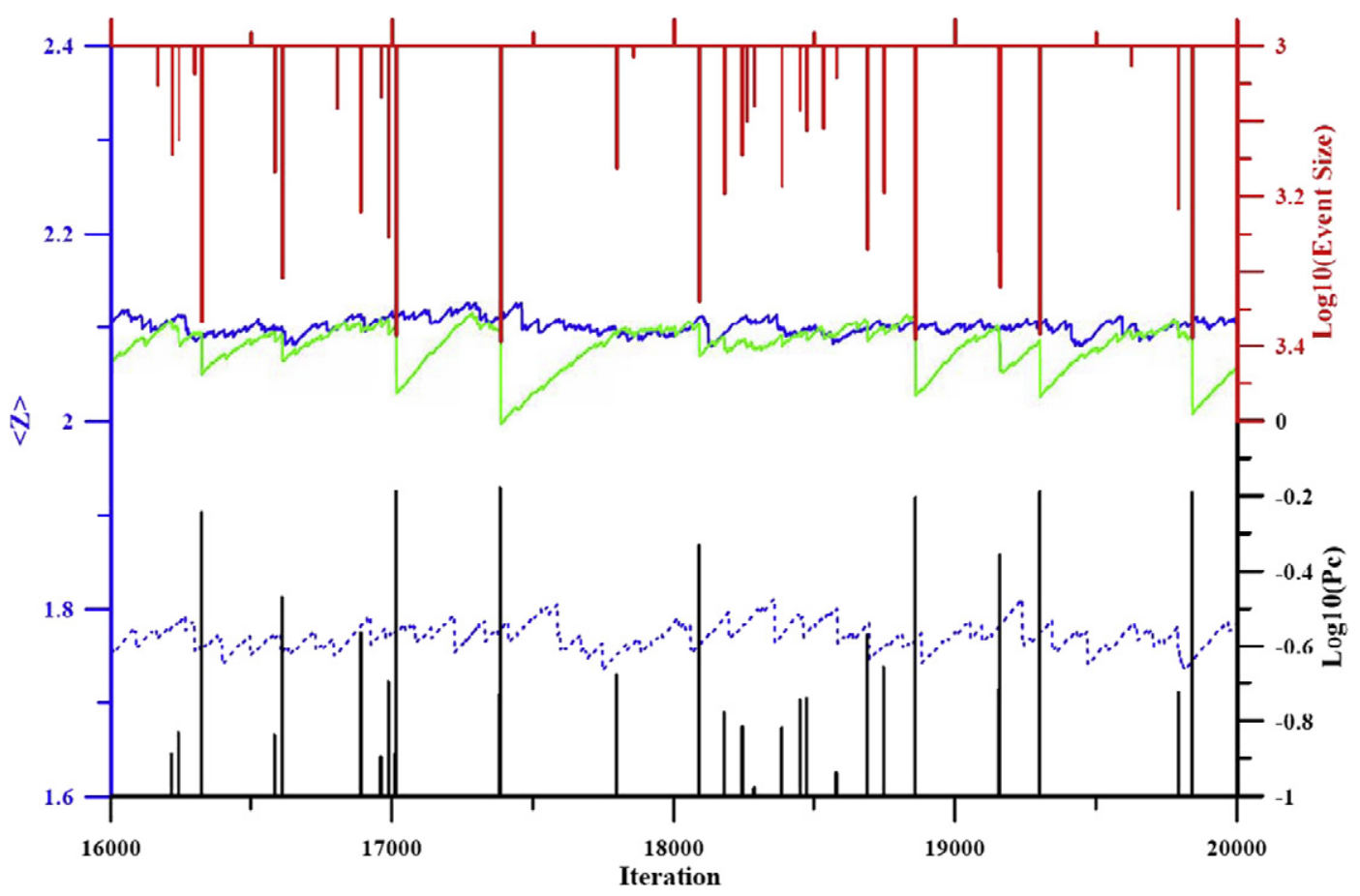

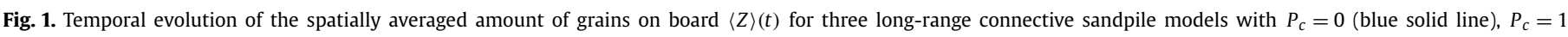

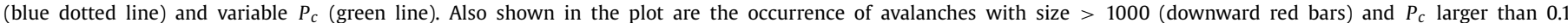

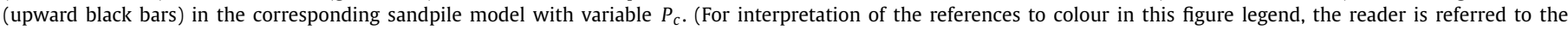
web version of this Letter.)

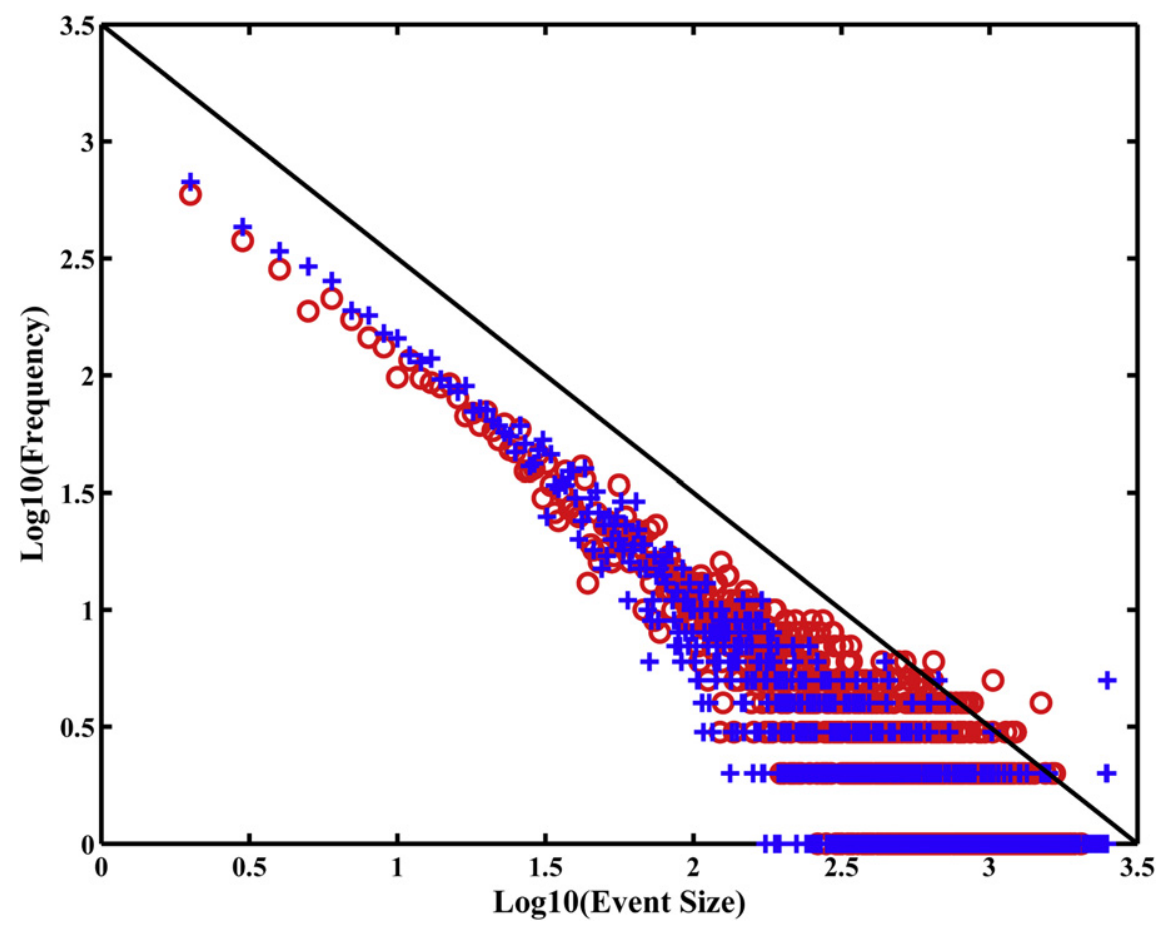

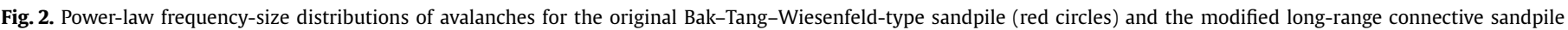

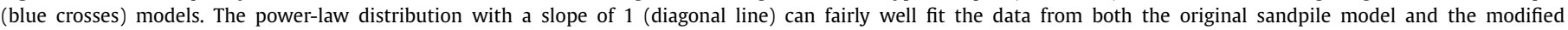

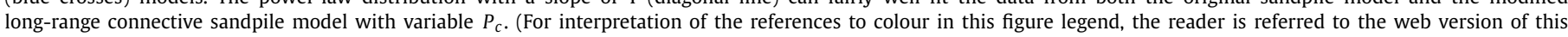
Letter.)

frequency-size power-law distribution, is established solely because of the dynamical interactions among individual elements of the system. Lomnitz-Adler [21] examined 40 variants of cellular automata focusing on their capability to reproduce a frequencysize power-law distribution.
Debating the question of continuous vs. discontinuous criticality for the earthquake fault system, the work of Sammis and Smith [11] is representative of a new class of models which display intermittent criticality. They built cellular automata with discrete fractal hierarchies and studied the effect of a loss factor, the $q$ 
value in their paper, on the behavior of the system. Such structurally heterogeneous and lossy $(q<1)$ automata produce large stress perturbations that move the system out of the critical state. In those models, a large event is followed by a shadow period of quiescence and then a new approach back toward the critical state. In another work, with regard to the effects of initial grid configuration, loading function, local dissipation and local redistribution, Castellaro and Mulargia [13] analyzed six different cellular automata. By means of involving a larger number of neighbors than four nearest neighbors, they found that the number of large events increases as approaching the main event. They thus claimed their finding supports that the Earth's crust approaches and retreats from an apparently critical state. Also, Ben-Zion et al. [16] constructed a discrete model of a 2-D heterogeneous fault with realistic dynamic weakening embedded in a 3-D elastic half-space. They concluded, due to the appearance of the cyclical structures in their analyzed dynamical variables for the system, the evolution in their model $F$ may be characterized overall as intermittent criticality.

Note that, for demonstrating the scenario of intermittent criticality, all of those above-mentioned models invoked some prescribed parameters to tune the sandpile/automaton system (e.g., the rescale factor of the discrete hierarchical fractal automaton, the percentage of the local energy dissipation, or the dynamic weakening coefficient). Since we have found that the probability of the long-range connections is a key parameter to affect the slope of power-law distribution [17], we here further construct an evolution rule for the long-range connection probability $P_{c}$. In the present LRCS model the $P_{c}$ itself evolves as an explicit function of the size of the latest event. Consequently, as $P_{c}$ changes, our model can display a similar character of intermittent criticality in the above-mentioned models $[11,13,16]$. As shown in Fig. 1, the energy decreases as a large event occurs. The system accordingly moves out of the critical state and then gets back toward the critical state waiting for next large event. To our knowledge, our LRCS mode thus represents a family of models with self-evolved intermittent criticality for simulating seismicity.

\section{Conclusion}

In this Letter we have proposed an alternative variant of sandpile models being able to demonstrate the intermittent criticality through adaptable long-range connections. The energy development, i.e., $\langle Z\rangle(t)$, in our LRCS model with variable $P_{c}$ mimics very much the evolution in the strain energy in the real earthquake fault system [24-27]. Since many researches $[3,12,14,28]$ have suggested that the earthquake fault system is not a system with the self-organized criticality but one with the intermittent criticality instead, the LRCS model with adaptable $P_{c}$ represents a type of simple and realistic sandpile model for simulating seismicity. It seems to us that the concept of long-range connection should be integrated into the numerical simulation process of seismicity. Moreover, many variables for detecting precursory phenomena of a forthcoming great event have been used in the literature [14-16,28]. It will be highly interesting to perform those measures for a synthetic catalogue generated by our LRCS model. Such a task will be very fundamental to the issue of earthquake prediction, and will be in detail presented elsewhere in the near future.

\section{Acknowledgements}

C.C.C. is grateful for research support from both the National Science Council (ROC) and the Institute of Geophysics (NCU, ROC). L.Y.C. is supported by the National Science Council of the Republic of China under grant NSC 96-2611-M-002-001. Thanks are also extended to one anonymous reviewer for improving the manuscript.

\section{References}

[1] A. Sornette, D. Sornette, Tectonophysics 179 (1990) 327.

[2] I. Main, Rev. Geophys. 34 (1996) 433.

[3] J.B. Rundle, W. Klein, D.L. Turcotte, B.D. Malamud, Pure Appl. Geophys. 157 (2000) 2165.

[4] G. Zoller, S. Hainzl, J. Kurths, J. Geophys. Res. B 106 (2001) 2167.

[5] J.B. Rundle, D.L. Turcotte, R. Shcherbakov, W. Klein, C. Sammis, Rev. Geophys. 41 (2003), doi:10.1029/2003RG000135.

[6] C.C. Chen, J.B. Rundle, H.C. Li, J.R. Holliday, D.L. Turcotte, K.F. Tiampo, Geophys. Res. Lett. 33 (2006), doi:10.1029/2006GL027323.

[7] P. Bak, C. Tang, K. Wiesenfeld, Phys. Rev. Lett. 59 (1987) 381.

[8] P. Bak, C. Tang, J. Geophys. Res. 94 (1989) 15635.

[9] A. Sornette, D. Sornette, Europhys. Lett. 9 (1989) 197

[10] K. Ito, M. Matsuzaki, J. Geophys. Res. 95 (1990) 6853.

[11] C.G. Sammis, S.W. Smith, Pure Appl. Geophys. 155 (1999) 307.

[12] J.B. Rundle, W. Klein, S. Gross, Pure Appl. Geophys. 155 (1999) 575.

[13] S. Castellaro, F. Mulargia, Geophys. J. Int. 150 (2002) 483.

[14] I.G. Main, F.H. Al-Kindy, Geophys. Res. Lett. 29 (2002), doi:10.1029/ $2001 \mathrm{GL} 014078$.

[15] D.D. Bowman, C.G. Sammis, Pure Appl. Geophys. 161 (2004) 1945.

[16] Y. Ben-Zion, M. Eneva, Y. Liu, J. Geophys. Res. B 108 (2003), doi:10.1029/ $2002 J B 002121$.

[17] C.C. Chen, L.Y. Chiao, Y.T. Lee, H.W. Cheng, Y.M. Wu, Tectonophysics (2008), 10.1016/j.tecto.2008.04.004.

[18] D.J. Watts, S.H. Strogatz, Nature 393 (1998) 440.

[19] P. Bak, How Nature Works: The Science of Self-Organized Criticality, Springer, New York, 1996.

[20] H.J. Jensen, Self-Organized Criticality: Emergent Complex Behavior in Physical and Biological Systems, Cambridge Univ. Press, Cambridge, 1998.

[21] J. Lomnitz-Adler, J. Geophys. Res. B 98 (1993) 17745.

[22] S. Castellaro, F. Mulargia, Geophys. J. Int. 150 (2001) 483.

[23] D. Weatherley, P. Mora, M.F. Xia, Pure Appl. Geophys. 159 (2002) 2469.

[24] K. Shimazaki, T. Nakata, Geophys. Res. Lett. 7 (1980) 279.

[25] C. Lomnitz, Fundamentals of Earthquake Prediction, Wiley, New York, 1994.

[26] F. Mulargia, P. Gasperini, Geophys. J. Int. 120 (1995) 453.

[27] H. Kanamori, E.E. Brodsky, Rep. Prog. Phys. 67 (2004) 1429

[28] C. Goltz, M. Bose, Geophys. Res. Lett. 29 (2002), doi:10.1029/2002GL015540. 